\title{
Chiral Cryptands Possessing Tetraazamacrocyclic and BINAM Moieties: Synthesis and Evaluation as Fluorescent Detectors
}

\author{
Olga K. Grigorova, ${ }^{a}$ Alexei D. Averin, ${ }^{\mathrm{a}, \mathrm{b} @ ~ O l g a ~ A . ~ M a l o s h i t s k a y a, ~}{ }^{a}{ }$ Franck Denat $^{\mathrm{c}}$ \\ and Irina P. Beletskaya ${ }^{\mathrm{a}, \mathrm{b}}$ \\ ${ }^{a}$ M.V. Lomonosov Moscow State University, Department of Chemistry, 119991 Moscow, Russia \\ ${ }^{\mathrm{b}}$ A.N. Frumkin Institute of Physical Chemistry and Electrochemistry RAS, 119991 Moscow, Russia \\ 'Institut de Chimie Moléculaire de l'Université de Bourgogne, Université de Bourgogne Franche-Comté, UMR CRNS $n^{\circ}$ \\ 6302, BP 47870, Dijon 21078 CEDEX, France \\ @Corresponding author E-mail: alexaveron@yandex.ru
}

\begin{abstract}
Pd(0)-Catalyzed amination of $N, N^{\prime}$-di(bromobenzyl) substituted tetraazamacrocycles (cyclen, cyclam) with (S)-2,2'diamino-1,1'-binaphthalene (BINAM) was employed for the synthesis of a novel type of chiral cryptands. Better yields were obtained for cyclen derivatives. The compounds were modified with dansyl groups to enhance their fluorescent properties. Cryptands theirselves and their dansyl derivatives were evaluated for sensing enantiomers of 7 amino alcohols and 21 metal cations.
\end{abstract}

Keywords: Macrocycles, Pd catalysis, chirality, fluorescence, detection

\section{Хиральные криптанды, содержащие фрагменты тетраазамакроциклов и БИНАМа: синтез и оценка в качестве флуоресцентных Аетекторов}

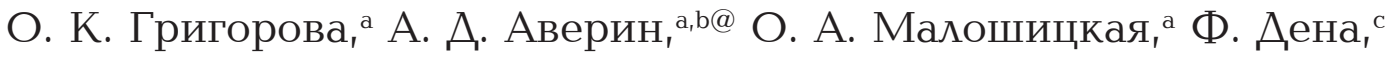 \\ И. П. Белецкая ${ }^{\mathrm{a}, \mathrm{b}}$
}

${ }^{a}$ Московский государственный университет им. М.В. Ломоносова, Химический факультет, 119991 Москва, Россия ${ }^{\mathrm{b}}$ Институт физической химии и электрохимии им. А.Н. Фрумкина, 119991 Москва, Россия

${ }^{\mathrm{c}}$ Институт молекулярной химии Университета Бургундии, Университет Бургундии Франш-Конте, 21078 Дижон, Франция

@E-mail: alexaveron@yandex.ru

Pd(0)-Катализируемое аминирование N,N'-ди(бромбензил)замещченных тетраазамакрочиклов (циклен, цчиклам) c (S)-2,2'-диамино-1,1'-бинафталином (БИНАМом) использовано для синтеза хиральньх криптандов нового типа. Более высокие выходы получены для производных ииклена. Полученные соединения модифицировали дансильными группами для улучшения флуоресиентных свойств соединений. Криптанды и их дансильные производные были исследованы в детектировании энантиомеров 7 аминоспиртов и 21 катиона металлов.

Ключевые слова: Макроциклы, Рd катализ, хиральность, флуоресценция, детектирование. 


\section{Introduction}

Fluorescent analytical methods are highly demanded due to their sensitivity, selectivity and wide opportunities of adjusting receptor and signaling units to certain analytes. Beginning from 1992, 1,1'-binaphthol (BINOL) has been employed as a basic moiety for creating chemosensors for fluorescence detection of chiral organic compounds. This is due to unique properties of BINOL: it combines $C 2$ chirality, fluorescent properties and possesses two oxygen atoms which can be employed in various structural modifications. ${ }^{[1]}$ BINOL derivatives were used mainly for sensing amino acids and their derivatives. For instance, 3,3'-disubstituted BINOLs were found to be useful for detecting $N$-Boc-alanine and $N$-Boc-phenylalanine by fluorescence quenching, ${ }^{[2,3]}$ emission enhancement was used for mandelic enantiomers sensing with a BINOL-diamine derivative. ${ }^{[4,5]}$ The same analyte can be detected by a macrocyclic sensor combining two BINOL and two chiral 1,2-diaminocyclohexane fragments. ${ }^{[6,7]}$ Macrocyclic derivatives of BINOL were also obtained employing imidazoline linkers, ${ }^{[8]}$ but in many cases BINOL was rather attached as an exocyclic structural fragment to other macrocycles such as triazacyclononane (sensor of tartrate anion), ${ }^{[9]}$ calix[4]crown ether, ${ }^{[10]}$ crown ethers containing additional exocyclic chiral groups. ${ }^{[1]}$ Additional chromophore ${ }^{[12]}$ and fluorophore ${ }^{[13]}$ groups were introduced in BINOL-crown ether conjugates to ensure better optical response. Even a cryptand possessing two BINOL and two coplanar porphyrins was contrived for better coordination. ${ }^{[14,15]}$

The analogue of BINOL, 2,2'-diamino-1,1'-binaphthalene (BINAM) was shown to be efficient for $\alpha$-phenylethylamine enantiomers sensing, ${ }^{[16]}$ however, unlike for BINOL, only scarce data can be found in literature considering its derivatives used as fluorescent detectors. ${ }^{[17-19]}$ We began our own investigations aimed at the synthesis of BINAMcontaining macrocycles and their use in fluorescent enantioselective detection of amino alcohols. ${ }^{[20-22]}$ The present work deals with the synthesis of cryptands on the basis of tetraazamacrocycles comprising endocyclic BINAM moiety.

\section{Experimental}

NMR spectra were registered using Bruker Avance 400 spectrometer, MALDI-TOF spectra were obtained with Bruker Autoflex II spectrometer using 1,8,9-trihydroxyanthracene as matrix and PEGs as internal standards. UV-Vis spectra were recorded with Agilent Cary 60 spectrophotometer in $\mathrm{MeCN}$, spectra of fluorescence were obtained with Hitachi 2700 spectrofluorometer in acetonitrile (UHPLC grade). (S)-2,2'diamino-1,1'-binaphthalene ((S)-BINAM) (5), 5-(dimethylamino) naphthalene-1-sulfonyl chloride (dansyl chloride) (11), BINAP ligand, optically active amino alcohols, sodium tert-butoxide, $\mathrm{Li}^{1}$, $\mathrm{Na}^{\mathrm{I}}, \mathrm{K}^{\mathrm{I}}, \mathrm{Mg}^{\mathrm{II}}, \mathrm{Ca}^{\mathrm{II}}, \mathrm{Ba}^{\mathrm{II}}, \mathrm{Al}^{\mathrm{III}}, \mathrm{Cr}^{\mathrm{III}}, \mathrm{Mn}^{\mathrm{II}}, \mathrm{Fe}^{\mathrm{II}}, \mathrm{Co}^{\mathrm{II}}, \mathrm{Ni}^{\mathrm{II}}, \mathrm{Cu}^{\mathrm{II}}, \mathrm{Zn}^{\mathrm{II}}$, $\mathrm{Cd}^{\text {II }}, \mathrm{Pb}^{\text {II }}, \mathrm{Ag}^{\mathrm{I}}, \mathrm{Hg}^{\mathrm{II}}$ (perchlorates), Ga ${ }^{\text {III }}, \mathrm{In}^{\text {III }}$, Y ${ }^{\mathrm{III}}$ (nitrates) were purchased from ABCR, Fluka and Sigma-Aldrich Co and used without further purification, $\mathrm{Pd}(\mathrm{dba})_{2}$ was synthesized according to the method described. ${ }^{[23]}$ Cyclen and cyclam derivatives 1-4 were obtained according to a method described in ref., ${ }^{[24,25]}$ using free cyclen and cyclam provided by CheMatech Co. Column chromatography was carried out using silica gel 40-63 nm
(Fluka). Acetonitrile of UHPLC grade was used without additional purification, dioxane was successively distilled over $\mathrm{NaOH}$ and sodium. Dichloromethane was distilled over $\mathrm{CaH}_{2}$, methanol was used freshly distilled.

Method for the synthesis of macrobicycles 6-9. A two-neck flask equipped with a magnetic stirrer and reflux condenser, flushed with dry argon, was charged with $\operatorname{Pd}(\mathrm{dba})_{2}(9 \mathrm{mg}, 0.016 \mathrm{mmol})$, BINAP (11 mg, $0.018 \mathrm{mmol}),(S)$-BINAM $5(0.2 \mathrm{mmol}, 57 \mathrm{mg})$, corresponding $N, N$ '-di(bromobenzyl) substituted cyclen or cyclam $(0.2 \mathrm{mmol}, 102 \mathrm{mg}$ or $107 \mathrm{mg})$, absolute dioxane $(8 \mathrm{ml})$ and sodium tert-butoxide $(0.6 \mathrm{mmol}, 58 \mathrm{mg})$. The reaction mixture was stirred under reflux for $24 \mathrm{~h}$, cooled to ambient temperature, solution was filtered, residue was washed with $10 \mathrm{ml}$ of dichloromethane, combined organic phases were evaporated in vacuo, and the residue was chromatographed on silica gel using a sequence of eluents: $\mathrm{CH}_{2} \mathrm{Cl}_{2}, \mathrm{CH}_{2} \mathrm{Cl}_{2}-\mathrm{MeOH} \quad 200: 1-3: 1, \quad \mathrm{CH}_{2} \mathrm{Cl}_{2}-\mathrm{MeOH}-\mathrm{NH}_{3}$ (aq) 100:20:1-100:20:3.

Cryptand 6. Eluent: $\mathrm{CH}_{2} \mathrm{Cl}_{2}-\mathrm{MeOH}$ 10:1 - 3:1. Yield $29 \mathrm{mg}$ (23\%), yellowish solid. (MALDI-TOF) found: 633.3671. $\mathrm{C}_{42} \mathrm{H}_{45} \mathrm{~N}_{6}$ requires $633.3706[\mathrm{M}+\mathrm{H}]^{+}$. ${ }^{1} \mathrm{H} \mathrm{NMR}\left(\mathrm{CDCl}_{3}, 298 \mathrm{~K}\right) \delta_{\mathrm{H}} \mathrm{ppm}$ : 2.41-2.47 m (2H), 2.58-2.75 m (6H), 2.83-2.93 m (6H), 2.94-3.02 $\mathrm{m}(2 \mathrm{H}), 3.57 \mathrm{~d}\left(2 \mathrm{H},{ }^{2} J=12.8 \mathrm{~Hz}\right), 3.69 \mathrm{~d}\left(2 \mathrm{H},{ }^{2} J=12.8 \mathrm{~Hz}\right), 5.76$ $\mathrm{s}(2 \mathrm{H}), 6.74 \mathrm{~d}\left(2 \mathrm{H},{ }^{3} J=7.6 \mathrm{~Hz}\right), 6.99 \mathrm{~d}\left(2 \mathrm{H},{ }^{3} J=8.1 \mathrm{~Hz}\right), 7.07-7.10$ $\mathrm{m}(4 \mathrm{H}), 7.14 \mathrm{t}\left(2 \mathrm{H},{ }^{3} \mathrm{~J}=7.8 \mathrm{~Hz}\right), 7.20-7.24 \mathrm{~m}(2 \mathrm{H}), 7.28-7.32 \mathrm{~m}$ $(2 \mathrm{H}), 7.73 \mathrm{~d}\left(2 \mathrm{H},{ }^{3} \mathrm{~J}=9.0 \mathrm{~Hz}\right), 7.84 \mathrm{~d}\left(2 \mathrm{H},{ }^{3} \mathrm{~J}=7.8 \mathrm{~Hz}\right), 7.91 \mathrm{~d}(2 \mathrm{H}$, $\left.{ }^{3} \mathrm{~J}=9.0 \mathrm{~Hz}\right)$, two $\mathrm{NH}$ protons were not assigned. ${ }^{13} \mathrm{C} \mathrm{NMR}\left(\mathrm{CDCl}_{3}\right.$, $298 \mathrm{~K}) \delta_{\mathrm{c}}$ ppm: 48.2 (2C), 48.5 (2C), 51.8 (2C), 52.6 (2C), 62.9 (2C), 116.3 (2C), $117.0(2 \mathrm{C}), 118.8$ (2C), $119.6(2 \mathrm{C}), 123.2(2 \mathrm{C})$, 123.5 (2C), 124.5 (2C), 127.1 (2C), 128.1 (2C), 129.2 (2C), 129.3 (2C), 129.5 (2C), 134.0 (2C), 139.6 (2C), 140.0 (2C), 142.5 (2C).

Macrotricycle 10. Obtained as the second product in the synthesis of the cryptand 6. Eluent: $\mathrm{CH}_{2} \mathrm{Cl}_{2}-\mathrm{MeOH}-$ $\mathrm{NH}_{3}$ (aq) 100:20:1. Yield $17 \mathrm{mg}$ (13\%), yellowish solid. (MALDITOF) found: 1265.7268. $\mathrm{C}_{84} \mathrm{H}_{89} \mathrm{~N}_{12}$ requires $1265.7333[\mathrm{M}+\mathrm{H}]^{+}$ ${ }^{1} \mathrm{H}$ NMR $\left(\mathrm{CDCl}_{3}, 298 \mathrm{~K}\right) \delta_{\mathrm{H}}$ ppm: $2.40-2.70 \mathrm{~m}(32 \mathrm{H}), 3.36 \mathrm{~d}(4 \mathrm{H}$, $\left.{ }^{2} J=13.0 \mathrm{~Hz}\right), 3.46 \mathrm{~d}\left(4 \mathrm{H},{ }^{2} J=13.0 \mathrm{~Hz}\right), 5.50 \mathrm{~s}(4 \mathrm{H}), 6.64 \mathrm{br} . \mathrm{d}(4 \mathrm{H}$, $\left.{ }^{3} J_{o b s}=7.0 \mathrm{~Hz}\right), 6.74-6.80 \mathrm{~m}(8 \mathrm{H}), 6.99 \mathrm{t}\left(4 \mathrm{H},{ }^{3} \mathrm{~J}=7.7 \mathrm{~Hz}\right), 7.12 \mathrm{~d}$ $\left(4 \mathrm{H},{ }^{3} J=8.2 \mathrm{~Hz}\right), 7.15-7.20 \mathrm{~m}(4 \mathrm{H}), 7.22-7.26 \mathrm{~m}(4 \mathrm{H}), 7.56 \mathrm{~d}(4 \mathrm{H}$, $\left.{ }^{3} J=9.0 \mathrm{~Hz}\right), 7.80 \mathrm{~d}\left(4 \mathrm{H},{ }^{3} J=8.5 \mathrm{~Hz}\right), 7.83 \mathrm{~d}\left(4 \mathrm{H},{ }^{3} J=9.0 \mathrm{~Hz}\right)$, four $\mathrm{NH}$ protons were not assigned. ${ }^{13} \mathrm{C} \mathrm{NMR}\left(\mathrm{CDCl}_{3}, 298 \mathrm{~K}\right) \delta_{\mathrm{c}} \mathrm{ppm}$ : 45.8 (8C), 51.8 (8C), 60.0 (4C), 116.0 (4C), 117.8 (4C), 119.2 (4C), 121.2 (4C), 123.1 (4C), 123.4 (4C), 124.3 (4C), 127.0 (4C), 128.2 (4C), 129.1 (4C), 129.3 (4C), 129.4 (4C), 133.8 (4C), 140.1 (4C), 140.8 (4C), 142.5 (4C).

Cryptand 7. Eluent: $\mathrm{CH}_{2} \mathrm{Cl}_{2}-\mathrm{MeOH}-\mathrm{NH}_{3}(\mathrm{aq})$ 100:20:1. Yield $44 \mathrm{mg}$ (34\%), yellowish solid. (MALDI-TOF) found: 633.38. $\mathrm{C}_{42} \mathrm{H}_{45} \mathrm{~N}_{6}$ requires $633.37[\mathrm{M}+\mathrm{H}]^{+}$; found: 631.3593 . $\mathrm{C}_{42} \mathrm{H}_{43} \mathrm{~N}_{6}$ requires $631.3549\left[\mathrm{M}-\mathrm{H}_{2}+\mathrm{H}\right]^{+}$; found: 629.3435. $\mathrm{C}_{42} \mathrm{H}_{41} \mathrm{~N}_{6}$ requires $629.3393\left[\mathrm{M}-2 \mathrm{H}_{2}+\mathrm{H}\right]^{+}$. ${ }^{2} \mathrm{H} \mathrm{NMR}\left(\mathrm{CDCl}_{3}, 298 \mathrm{~K}\right) \delta_{\mathrm{H}} \mathrm{ppm}$ : $2.35-2.50 \mathrm{~m}(2 \mathrm{H}), 2.51-2.65 \mathrm{~m}(6 \mathrm{H}), 2.65-2.80 \mathrm{~m}(8 \mathrm{H}), 3.40 \mathrm{~d}$ $\left(2 \mathrm{H},{ }^{2} J=14.0 \mathrm{~Hz}\right), 3.54 \mathrm{~d}\left(2 \mathrm{H},{ }^{2} J=14.0 \mathrm{~Hz}\right), 4.66$ br.s $(2 \mathrm{H}), 5.58$ $\mathrm{s}(2 \mathrm{H}), 6.73 \mathrm{~d}\left(4 \mathrm{H},{ }^{3} J=8.3 \mathrm{~Hz}\right), 6.96 \mathrm{~d}\left(4 \mathrm{H},{ }^{3} J=8.3 \mathrm{~Hz}\right), 7.24 \mathrm{~d}$ $\left(2 \mathrm{H},{ }^{3} J=7.8 \mathrm{~Hz}\right), 7.29-7.33 \mathrm{~m}(2 \mathrm{H}), 7.35-7.39 \mathrm{~m}(2 \mathrm{H}), 7.48 \mathrm{~d}(2 \mathrm{H}$, $\left.{ }^{3} J=8.9 \mathrm{~Hz}\right), 7.85-7.88 \mathrm{~m}(4 \mathrm{H}) .{ }^{13} \mathrm{C} \mathrm{NMR}\left(\mathrm{CDCl}_{3}, 298 \mathrm{~K}\right) \delta_{\mathrm{c}} \mathrm{ppm}$ : $46.8(2 \mathrm{C}), 51.3(2 \mathrm{C}), 52.4(2 \mathrm{C}), 52.6(2 \mathrm{C}), 61.1(2 \mathrm{C}), 120.2(2 \mathrm{C})$, 120.9 (4C), $123.8(2 \mathrm{C}), 124.6(2 \mathrm{C}), 127.1(2 \mathrm{C}), 128.3(2 \mathrm{C}), 129.3$ (8C), 129.7 (2C), 133.9 (2C), 134.0 (2C), 140.6 (2C), 142.4 (2C).

Cryptand 8. Eluent: $\mathrm{CH}_{2} \mathrm{Cl}_{2}-\mathrm{MeOH}-\mathrm{NH}_{3}(\mathrm{aq})$ 100:20:3 Yield $22 \mathrm{mg}$ (16\%), yellowish solid. (MALDI-TOF) found: 661.3967. $\mathrm{C}_{44} \mathrm{H}_{49} \mathrm{~N}_{6}$ requires $661.4019[\mathrm{M}+\mathrm{H}]^{+} .{ }^{1} \mathrm{H} \mathrm{NMR}\left(\mathrm{CDCl}_{3}\right.$, $298 \mathrm{~K}) \delta_{\mathrm{H}}$ ppm: $1.58-1.66 \mathrm{~m}(1 \mathrm{H}), 1.66-1.78 \mathrm{~m}(2 \mathrm{H}), 1.78-1.86$ $\mathrm{m}(1 \mathrm{H}), 2.23-2.74 \mathrm{~m}(12 \mathrm{H}), 2.81-2.96 \mathrm{~m}(3 \mathrm{H}), 3.02 \mathrm{~d}(1 \mathrm{H}$, $\left.{ }^{2} J=14.0 \mathrm{~Hz}\right), 3.05-3.09 \mathrm{~m}(1 \mathrm{H}), 3.13 \mathrm{~d}\left(1 \mathrm{H},{ }^{2} J=14.0 \mathrm{~Hz}\right), 3.57$ $\mathrm{d}\left(1 \mathrm{H},{ }^{2} J=14.0 \mathrm{~Hz}\right), 3.83 \mathrm{~d}\left(1 \mathrm{H},{ }^{2} J=14.0 \mathrm{~Hz}\right), 5.75 \mathrm{~s}(1 \mathrm{H}), 5.81 \mathrm{~s}$ $(1 \mathrm{H}), 6.75 \mathrm{~d}\left(1 \mathrm{H},{ }^{3} J=7.6 \mathrm{~Hz}\right), 6.79 \mathrm{~d}\left(1 \mathrm{H},{ }^{3} \mathrm{~J}=7.5 \mathrm{~Hz}\right), 6.92 \mathrm{~d}(1 \mathrm{H}$, $\left.{ }^{3} J=7.8 \mathrm{~Hz}\right), 7.00 \mathrm{~d}\left(1 \mathrm{H},{ }^{3} J=8.2 \mathrm{~Hz}\right), 7.06-7.14 \mathrm{~m}(4 \mathrm{H}), 7.19-7.31 \mathrm{~m}$ $(6 \mathrm{H}), 7.51 \mathrm{~d}\left(1 \mathrm{H},{ }^{3} \mathrm{~J}=9.0 \mathrm{~Hz}\right), 7.62 \mathrm{~d}\left(1 \mathrm{H},{ }^{3} \mathrm{~J}=9.0 \mathrm{~Hz}\right), 7.79-7.84 \mathrm{~m}$ $(3 \mathrm{H}), 7.85 \mathrm{~d}\left(1 \mathrm{H},{ }^{3} J=9.0 \mathrm{~Hz}\right)$, two NH protons were not assigned. 
${ }^{13} \mathrm{C}$ NMR $\left(\mathrm{CDCl}_{3}, 298 \mathrm{~K}\right) \delta$ ppm: 25.6, 25.9, 46.8, 49.0, 49.8, 50.1, 51.0, 52.5, 54.6 (2C), 57.3, 57.7, 116.4, 117.5, 118.0 (2C), 119.1, $119.4,121.5,122.9,123.0,123.1,123.2,123.6,124.4,124.6,126.8$ (2C), 128.0, 128.1, 128.4, 128.8, 129.2 (3C), 129.3, 133.8, 134.1, 140.6, 140.8, 141.0, 141.7, 142.3, 142.7 .

Cryptand 9. Eluent: $\mathrm{CH}_{2} \mathrm{Cl}_{2}-\mathrm{MeOH}$ 3:1. Yield $18 \mathrm{mg}$ (13\%), yellowish solid. (MALDI-TOF) found: 661.4068. $\mathrm{C}_{44} \mathrm{H}_{49} \mathrm{~N}_{6}$ requires $661.4019[\mathrm{M}+\mathrm{H}]^{+} .{ }^{1} \mathrm{H} \mathrm{NMR}\left(\mathrm{CDCl}_{3}, 298 \mathrm{~K}\right) \delta_{\mathrm{H}} \mathrm{ppm}$ : $1.79-1.90 \mathrm{~m}(2 \mathrm{H}), 2.10-2.21 \mathrm{~m}(2 \mathrm{H}), 2.42-2.52 \mathrm{~m}(4 \mathrm{H}), 2.70-2.82$ $\mathrm{m}(6 \mathrm{H}), 2.81-2.93 \mathrm{~m}(6 \mathrm{H}), 3.09 \mathrm{~d}\left(2 \mathrm{H},{ }^{2} J=13.0 \mathrm{~Hz}\right), 3.66 \mathrm{~d}(2 \mathrm{H}$, $\left.{ }^{2} J=13.0 \mathrm{~Hz}\right), 5.32 \mathrm{~s}(2 \mathrm{H}), 6.89 \mathrm{~d}\left(4 \mathrm{H},{ }^{3} J=8.1 \mathrm{~Hz}\right), 6.93 \mathrm{~d}(4 \mathrm{H}$, $\left.{ }^{3} J=8.1 \mathrm{~Hz}\right), 7.20 \mathrm{~d}\left(2 \mathrm{H},{ }^{3} J=8.1 \mathrm{~Hz}\right), 7.29-7.33 \mathrm{~m}(2 \mathrm{H}), 7.36-7.40$ $\mathrm{m}(2 \mathrm{H}), 7.42 \mathrm{~d}\left(2 \mathrm{H},{ }^{3} J=9.0 \mathrm{~Hz}\right), 7.84-7.89 \mathrm{~m}(4 \mathrm{H})$, two NH protons were not assigned.

\section{Dansyl-substituted cryptands:}

Cryptand 12. A flask equipped with a magnetic stirrer was charged with the cryptand $6(0.03 \mathrm{mmol}, 19 \mathrm{mg})$, dansyl chloride 11 (0.066 mmol, $19 \mathrm{mg}), \mathrm{K}_{2} \mathrm{CO}_{3}(0.12 \mathrm{mmol}, 18 \mathrm{mg})$ and $1 \mathrm{ml} \mathrm{MeCN}$. The reaction mixture was stirred at ambient temperature for $24 \mathrm{~h}$, the residue was filtered, washed with $5 \mathrm{ml}$ dichloromethane, combined organic fractions were evaporated in vacuo, the residue was dissolved in dichloromethane $(3 \mathrm{ml})$, washed with water $(3 \times 5 \mathrm{ml})$, dried over molecular sieves $4 \AA$, evaporated in vacuo, and the target compound $\mathbf{1 2}$ was obtained as a yellow glassy compound. Yield $30 \mathrm{mg}$ (90\%). (MALDI-TOF) found: 1099.4802. $\mathrm{C}_{66} \mathrm{H}_{67} \mathrm{~N}_{8} \mathrm{O}_{4} \mathrm{~S}_{2}$ requires $1099.4727[\mathrm{M}+\mathrm{H}]^{+}$. ${ }^{1} \mathrm{H}$ NMR $\left(\mathrm{CDCl}_{3}, 298 \mathrm{~K}\right) \delta_{\mathrm{H}}$ ppm: $2.60-2.71 \mathrm{~m}(6 \mathrm{H}), 2.80-2.91 \mathrm{~m}$ $(4 \mathrm{H}), 2.81 \mathrm{~s}(12 \mathrm{H}), 3.22 \mathrm{~d}\left(2 \mathrm{H},{ }^{2} J=13.4 \mathrm{~Hz}\right), 3.25-3.31 \mathrm{~m}(2 \mathrm{H})$, 3.34 br.t $\left(4 \mathrm{H},{ }^{3} \mathrm{~J}=5.4 \mathrm{~Hz}\right), 3.45 \mathrm{~d}\left(2 \mathrm{H},{ }^{2} J=13.4 \mathrm{~Hz}\right), 5.66 \mathrm{~s}(2 \mathrm{H})$, $6.63 \mathrm{~d}\left(2 \mathrm{H},{ }^{3} \mathrm{~J}=7.6 \mathrm{~Hz}\right), 6.90 \mathrm{~d}\left(2 \mathrm{H},{ }^{3} \mathrm{~J}=8.1 \mathrm{~Hz}\right), 6.96 \mathrm{~d}(2 \mathrm{H}$, $\left.{ }^{3} J=7.5 \mathrm{~Hz}\right), 7.03 \mathrm{t}\left(2 \mathrm{H},{ }^{3} J=7.8 \mathrm{~Hz}\right), 7.08$ br.s $(2 \mathrm{H}), 7.15 \mathrm{~d}(2 \mathrm{H}$, $\left.{ }^{3} J=7.8 \mathrm{~Hz}\right), 7.22-7.35 \mathrm{~m}(8 \mathrm{H}), 7.61 \mathrm{~d}(2 \mathrm{H}, 9.0 \mathrm{~Hz}), 7.79 \mathrm{dd}(2 \mathrm{H}$, $\left.{ }^{3} J=7.3 \mathrm{~Hz},{ }^{4} J=1.5 \mathrm{~Hz}\right), 7.85-7.89 \mathrm{~m}(4 \mathrm{H}), 8.17 \mathrm{~d}\left(2 \mathrm{H},{ }^{3} J=8.6 \mathrm{~Hz}\right)$, $8.41 \mathrm{~d}\left(2 \mathrm{H},{ }^{3} \mathrm{~J}=8.5 \mathrm{~Hz}\right) .{ }^{13} \mathrm{C} \mathrm{NMR}\left(\mathrm{CDCl}_{3}, 298 \mathrm{~K}\right) \delta_{\mathrm{c}} \mathrm{ppm}: 45.3$ (4C), 46.9 (2C), 47.4 (2C), 53.1 (2C), 54.6 (2C), 60.4 (2C), 115.0 (2C), 116.1 (2C), 117.7 (2C), 119.5 (2C), 120.0 (2C), 121.4 (2C), 122.9 (2C), 123.2 (2C), 123.4 (2C), 124.5 (2C), 126.9 (2C), 127.8 (2C), 128.2 (2C), 128.8 (2C), 129.1 (2C), 129.3 (2C), 129.4 (2C), 129.9 (2C), 130.1 (2C), 134.2 (2C), 134.5 (2C), 139.9 (2C), 140.7 (2C), 142.7 (2C), 151.5 (2C), two quaternary carbon atoms were not assigned.

Cryptand 13. A flask equipped with a magnetic stirrer was charged with the cryptand $7(0.06 \mathrm{mmol}, 38 \mathrm{mg})$, dansyl chloride 11 (0.132 mmol, $36 \mathrm{mg}), \mathrm{K}_{2} \mathrm{CO}_{3}(0.26 \mathrm{mmol}, 37 \mathrm{mg})$ and $1 \mathrm{ml} \mathrm{MeCN}$. The reaction mixture was stirred at ambient temperature for $24 \mathrm{~h}$, the residue was filtered, washed with $5 \mathrm{ml}$ dichloromethane, combined organic fractions were evaporated in vacuo, the residue was dissolved in dichloromethane $(3 \mathrm{ml})$, washed with water $(3 \times 5 \mathrm{ml})$, dried over molecular sieves $4 \AA$, evaporated in vacuo, and chromatographed on silica gel using a sequence of eluents: $\mathrm{CH}_{2} \mathrm{Cl}_{2}, \mathrm{CH}_{2} \mathrm{Cl}_{2}-\mathrm{MeOH}$ 200:1-10:1. Eluent: $\mathrm{CH}_{2} \mathrm{Cl}_{2}-\mathrm{MeOH}$ 100:1. Yield $20 \mathrm{mg}$ (30\%), yellow glassy compound. (MALDI-TOF) found: 1099.48. $\mathrm{C}_{66} \mathrm{H}_{67} \mathrm{~N}_{8} \mathrm{O}_{4} \mathrm{~S}_{2}$ requires $1099.47[\mathrm{M}+\mathrm{H}]^{+} .{ }^{1} \mathrm{H}$ NMR $\left(\mathrm{CDCl}_{3}, 298 \mathrm{~K}\right) \delta_{\mathrm{H}} \mathrm{ppm}: 2.55-2.63 \mathrm{~m}$ (2H), 2.66-2.75 m (4H), $2.77 \mathrm{~s} \mathrm{(12H),} \mathrm{2.82-2.90} \mathrm{m} \mathrm{(2H),} \mathrm{2.99-3.06}$ $\mathrm{m}(2 \mathrm{H}), 3.09 \mathrm{~d}\left(2 \mathrm{H},{ }^{2} J=13.6 \mathrm{~Hz}\right), 3.18-3.26 \mathrm{~m}(2 \mathrm{H}), 3.36-3.45 \mathrm{~m}$ $(4 \mathrm{H}), 3.58 \mathrm{~d}\left(2 \mathrm{H},{ }^{2} J=13.6 \mathrm{~Hz}\right), 5.24 \mathrm{~s}(2 \mathrm{H}), 6.46 \mathrm{~d}\left(4 \mathrm{H},{ }^{3} J=8.1 \mathrm{~Hz}\right)$, $6.75 \mathrm{~d}\left(4 \mathrm{H},{ }^{3} \mathrm{~J}=8.1 \mathrm{~Hz}\right), 6.87 \mathrm{~d}\left(2 \mathrm{H},{ }^{3} \mathrm{~J}=7.3 \mathrm{~Hz}\right), 7.17 \mathrm{~d}(2 \mathrm{H}$, $\left.{ }^{3} J=8.3 \mathrm{~Hz}\right), 7.28-7.36 \mathrm{~m}(6 \mathrm{H}), 7.42 \mathrm{t}\left(2 \mathrm{H},{ }^{3} J=7.4 \mathrm{~Hz}\right), 7.53 \mathrm{~d}(2 \mathrm{H}$, $\left.{ }^{3} J=8.8 \mathrm{~Hz}\right), 7.95 \mathrm{~d}\left(2 \mathrm{H},{ }^{3} J=8.1 \mathrm{~Hz}\right), 7.97 \mathrm{~d}\left(2 \mathrm{H},{ }^{3} J=9.0 \mathrm{~Hz}\right), 8.08 \mathrm{~d}$ $\left(2 \mathrm{H},{ }^{3} J=7.2 \mathrm{~Hz}\right), 8.23 \mathrm{~d}\left(2 \mathrm{H},{ }^{3} J=8.7 \mathrm{~Hz}\right), 8.32 \mathrm{~d}\left(2 \mathrm{H},{ }^{3} J=8.6 \mathrm{~Hz}\right)$. ${ }^{13} \mathrm{C} \mathrm{NMR}\left(\mathrm{CDCl}_{3}, 298 \mathrm{~K}\right) \delta_{\mathrm{c}} \mathrm{ppm}: 43.8(2 \mathrm{C}), 45.3(4 \mathrm{C}), 45.4(2 \mathrm{C})$, 51.9 (2C), 52.5 (2C), 60.1 (2C), 114.9 (2C), 118.1 (2C), 118.5 (4C), 119.5 (2C), 120.3 (2C), 123.0 (2C), 123.9 (2C), 124.7 (2C), 127.1 (2C), 127.9 (2C), 128.4 (2C), 129.3 (2C), 129.7 (2C), 129.8 (2C), 130.0 (2C), 130.1 (2C), 130.2 (4C), 131.8 (2C), 134.2 (2C), 134.3 (2C), 140.5 (2C), 142.2 (2C), 151.7 (2C), two quaternary carbon atoms were not assigned.
Cryptand 14. A flask equipped with a magnetic stirrer was charged with the cryptand $8(0.026 \mathrm{mmol}, 17 \mathrm{mg})$, dansyl chloride 11 ( $0.130 \mathrm{mmol}, 33 \mathrm{mg}), \mathrm{K}_{2} \mathrm{CO}_{3}(0.2 \mathrm{mmol}, 28 \mathrm{mg})$ and $1 \mathrm{ml}$ $\mathrm{MeCN}$. The reaction mixture was stirred at ambient temperature for $24 \mathrm{~h}$, the residue was filtered, washed with $5 \mathrm{ml}$ dichloromethane, combined organic fractions were evaporated in vacuo, the residue was dissolved in dichloromethane $(3 \mathrm{ml})$, washed with water $(3 \times 5 \mathrm{ml})$, dried over molecular sieves $4 \AA$, evaporated in vacuo, and chromatographed on silica gel using a sequence of eluents: $\mathrm{CH}_{2} \mathrm{Cl}_{2}, \mathrm{CH}_{2} \mathrm{Cl}_{2}-\mathrm{MeOH}$ 500:1-50:1. Eluent: $\mathrm{CH}_{2} \mathrm{Cl}_{2}-\mathrm{MeOH}$ 500:1. Yield $20 \mathrm{mg}$ (69\%), yellow glassy compound. (MALDI-TOF) found: 1127.5098. $\mathrm{C}_{68} \mathrm{H}_{71} \mathrm{~N}_{8} \mathrm{O}_{4} \mathrm{~S}_{2}$ requires $1127.5040[\mathrm{M}+\mathrm{H}]^{+} .{ }^{1} \mathrm{H}$ NMR $\left(\mathrm{CDCl}_{3}, 298 \mathrm{~K}\right) \delta_{\mathrm{H}} \mathrm{ppm}: 1.60-1.68 \mathrm{~m}(2 \mathrm{H}), 2.00-2.05 \mathrm{~m}$ (2H), $2.14 \mathrm{dt}\left(2 \mathrm{H},{ }^{2} J=13.4 \mathrm{~Hz},{ }^{3} J=4.4 \mathrm{~Hz}\right), 2.29-2.36 \mathrm{~m}(2 \mathrm{H})$, $2.40 \mathrm{~s}(12 \mathrm{H}), 2.52 \mathrm{~d}\left(2 \mathrm{H},{ }^{2} J=12.9 \mathrm{~Hz}\right), 2.56 \mathrm{~d}\left(2 \mathrm{H},{ }^{2} J=12.9 \mathrm{~Hz}\right)$, $2.83-2.90 \mathrm{~m}(4 \mathrm{H}), 3.32-3.37 \mathrm{~m}(4 \mathrm{H}), 3.40-3.49 \mathrm{~m}(2 \mathrm{H}), 3.64-3.73$ $\mathrm{m}(2 \mathrm{H}), 5.67 \mathrm{~s}(2 \mathrm{H}), 5.89 \mathrm{~d}\left(2 \mathrm{H},{ }^{3} J=7.6 \mathrm{~Hz}\right), 6.25-6.29 \mathrm{~m}(4 \mathrm{H})$, $6.43 \mathrm{~d}\left(2 \mathrm{H},{ }^{3} J=7.6 \mathrm{~Hz}\right), 7.01 \mathrm{t}\left(2 \mathrm{H},{ }^{3} J=7.6 \mathrm{~Hz}\right), 7.11-7.17 \mathrm{~m}(4 \mathrm{H})$, $7.27-7.33 \mathrm{~m}(6 \mathrm{H}), 7.77 \mathrm{~d}\left(2 \mathrm{H},{ }^{3} J=9.0 \mathrm{~Hz}\right), 7.82 \mathrm{~d}\left(2 \mathrm{H},{ }^{3} J=7.6 \mathrm{~Hz}\right)$, $7.91-7.96 \mathrm{~m}(4 \mathrm{H}), 8.02 \mathrm{~d}\left(2 \mathrm{H},{ }^{3} \mathrm{~J}=8.9 \mathrm{~Hz}\right), 8.06 \mathrm{~d}\left(2 \mathrm{H},{ }^{3} \mathrm{~J}=8.3 \mathrm{~Hz}\right)$.

The investigations of the spectral properties of the cryptands 6-9 and 13 in the presence of different chiral molecules were carried out in a following manner: $3 \mathrm{ml}$ of the solution of $\mathbf{6}(C=31.0$ $\mu \mathrm{M})$, or $7(C=16.0 \mu \mathrm{M})$, or $\mathbf{8}(C=15.0 \mu \mathrm{M})$, or $\mathbf{9}(C=12.1 \mu \mathrm{M})$, or $\mathbf{1 3}(C=12.5 \mu \mathrm{M})$, in $\mathrm{MeCN}$ was placed in a spectrofluorimetric cuvette, solutions of appropriate chiral compounds $(S)$ - and $(R)$ leucinol, $(S)$ - and $(R)$-tert-leucinol, $(S)$ - and $(R)$-phenylglycinol, $(S)$ - and $(R)$-2-aminopropan-1-ol, $(S)$ - and $(R)$-2-aminobutan-1-ol, $(1 S, 2 R)$ - and $(1 R, 2 S)$-2-amino-1,2-diphenylethanol, in $\mathrm{MeCN}$ $(C=0.2 \mathrm{M})$ were added sequentially $(100,200,500,1000$ equiv. $)$ and after each addition UV-Vis and fluorescence spectra were recorded. Also the solutions of $\mathrm{Li}^{\mathrm{I}}, \mathrm{Na}^{\mathrm{I}}, \mathrm{K}^{\mathrm{I}}, \mathrm{Ag}^{\mathrm{I}}, \mathrm{Mg}^{\mathrm{II}}, \mathrm{Ca}^{\mathrm{II}}, \mathrm{Ba}^{\mathrm{II}}$, $\mathrm{Al}^{\mathrm{III}}, \mathrm{Fe}^{\mathrm{II}}, \mathrm{Mn}^{\text {II }}, \mathrm{Co}^{\mathrm{II}}, \mathrm{Ni}^{\text {II }}, \mathrm{Cr}^{\mathrm{III}}, \mathrm{Cu}^{\text {II }}, \mathrm{Zn}^{\text {II }}, \mathrm{Cd}^{\mathrm{II}}, \mathrm{Pb}^{\text {II }}, \mathrm{Hg}^{\text {II }}$ perchlorates and $\mathrm{Ga}^{\mathrm{III}}$, In ${ }^{\mathrm{III}}$, $\mathrm{Y}^{\mathrm{III}}$ nitrates in $\mathrm{MeCN}(C=0.01 \mathrm{M})$ were added sequentially to the solutions of the cryptands 6-9 and $\mathbf{1 2}$ in $\mathrm{MeCN}$ $(1,2$, and 5 equiv. $)$ and after each addition UV-Vis and fluorescence spectra were recorded.

\section{Results and Discussion}

The synthesis of the target cryptands was accomplished using Buchwald-Hartwig amination applied to the macrocyclization reactions. They were successfully employed by us earlier to obtain various macrobicyclic and macrotricyclic derivatives of cyclen (1,4,7,10-tetraazacyclododecane) and cyclam $\left(1,4,8,11\right.$-tetraazacyclotetradecane). ${ }^{[24-31]}$ The starting compounds, $N, N$ '-di(bromobenzyl) substituted cyclens 1, 2 and cyclams 3,4 were synthesized in high yields from free tetraazamacrocycles by three-step procedures described previously. ${ }^{[24,25]}$ The reactions of these compounds with $(S)$-BINAM (5) were carried out in the presence of the catalytic system Pd(dba) $)_{2}$ BINAP ( $8 / 9 \mathrm{~mol} \%$ ) in boiling dioxane at the concentration of the starting compounds $0.02 \mathrm{M}$ using sodium tert-butoxide as a base (Scheme 1). After the reactions were over, target cryptands were isolated from the reaction mixtures using column chromatography. This approach proved to be quite efficient in the present case as it provided the desired cryptands 6-9 in yields up to $34 \%$. It is to be noted, however, that the steric constraint in this cyclization reaction is quite strict due to the rigid structure of BINAM with a short distance between the two amino groups. It is not surprising that for different bromobenzyl derivatives of tetraazamacrocycles the outcome of the reaction was different. As usual, macrocyclization reaction 


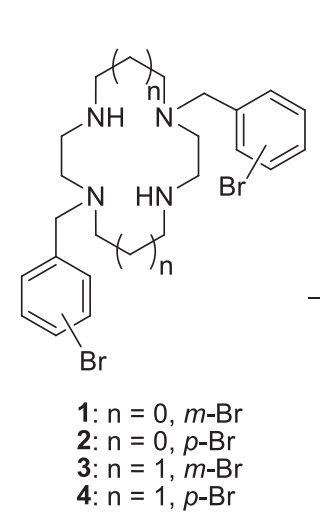<smiles>Nc1ccc(N)c(-c2c(N)ccc3ccccc23)c1</smiles>

5, 1 equiv

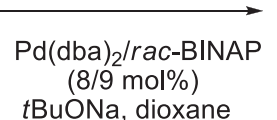
tBuONa, dioxane<smiles>Pc1ccccc1-c1ccc2ccccc2c1-c1c(P)ccc2ccccc12</smiles>

rac-BINAP

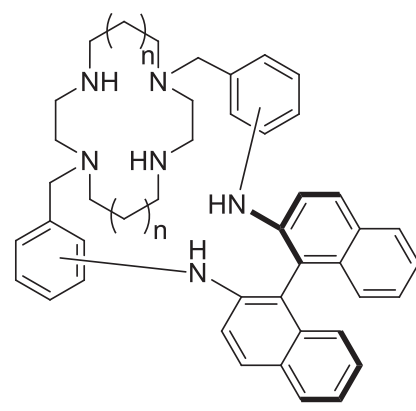

6: $\mathrm{n}=0, m-\mathrm{NH}, 23 \%$ 7: $\mathrm{n}=0, p-\mathrm{NH}, 34 \%$ 8: $\mathrm{n}=1, m-\mathrm{NH}, 16 \%$ 9: $\mathrm{n}=1, p-\mathrm{NH}, 13 \%$

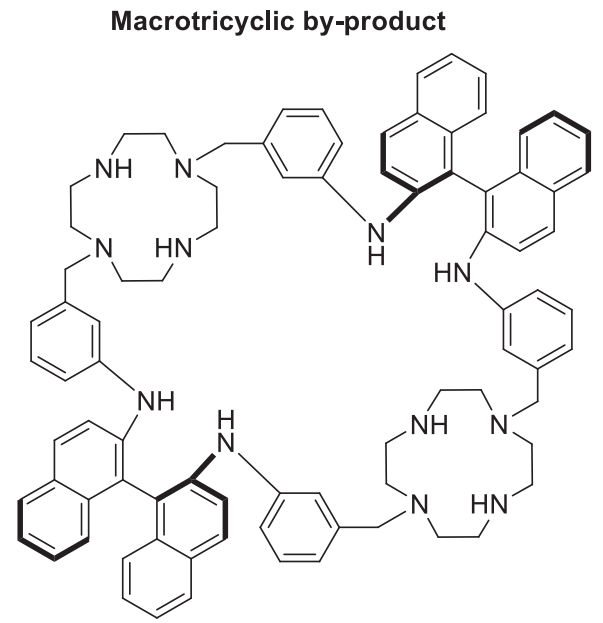

$10,13 \%$

Scheme 1.

proceeds in poorer yields in the cyclam series. In one case, namely with $N, N$ '-di(3-bromobenzyl)cyclen, we managed to isolate the cyclic dimer $\mathbf{1 0}$, actually a macrotricycle, as a by-product in $13 \%$ yield. In the same reaction a mixture of cyclic trimers and tetramer was also obtained as a separate fraction (yield $20 \%$ ). This compound is of interest as it possesses a larger macrocyclic cavity compared to the cryptands 6-9. In other cases, only fractioned mixture of cyclic and linear oligomers was obtained by chromatography.

Next, we modified the synthesized macrobicycles with dansyl fluorophore groups. For this purpose the cryptands 6-9 were reacted with 2.2 equiv. of 5 -(dimethylamino) naphthalene-1-sulfonyl chloride (dansyl chloride 11) in $\mathrm{MeCN}$ in the presence of $\mathrm{K}_{2} \mathrm{CO}_{3}$ at ambient temperature (Scheme 2). Macrobicycles 6-8 produced corresponding didansyl derivatives $\mathbf{1 2 - 1 4}$ in yields from 30 to $90 \%$, while compound 9 was unable to afford the target product. Such difference in the reactivity of the cryptands is supposedly due to the difference in the steric hindrances at the secondary amino groups of the tetraazamacrocycles.
Indeed, quite close position of the binaphthalene moiety to the tetraazamacrocycle may hinder the alkylation of these amino groups with naphthalene-1-sulfonyl chloride. Didansyl derivatives $\mathbf{1 3}$ and $\mathbf{1 4}$ were isolated using column chromatography on silica gel.

NMR spectra of the obtained cryptands possess the following peculiarities. In all macrobicycles protons in $\mathrm{NCH}_{2} \mathrm{Ph}$ methylene group are diastereotopic. Cyclen fragment in compounds 6, 7, 12 and $\mathbf{1 3}$ is characterized by four different carbon atoms and not two as was usual for many other achiral macrobicycles synthesized by us earlier. However, macrotricyclic dimer $\mathbf{1 0}$ displays only two different carbon atoms assigned for cyclen moiety which can be explained by a different reciprocal position of cyclen and BINAM fragments. In the case of cyclam derivative $\mathbf{8}$ the asymmetrical effect is even more pronounced as all protons and carbons in this molecule are inequivalent thus leading to a complicated spectral pattern. On the other hand, in other cyclam derivatives (9 and 14) symmetry is not disturbed, benzyl spacers and naphthalene moieties

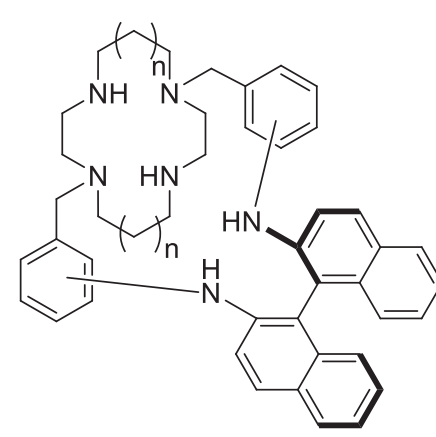

$6-8$

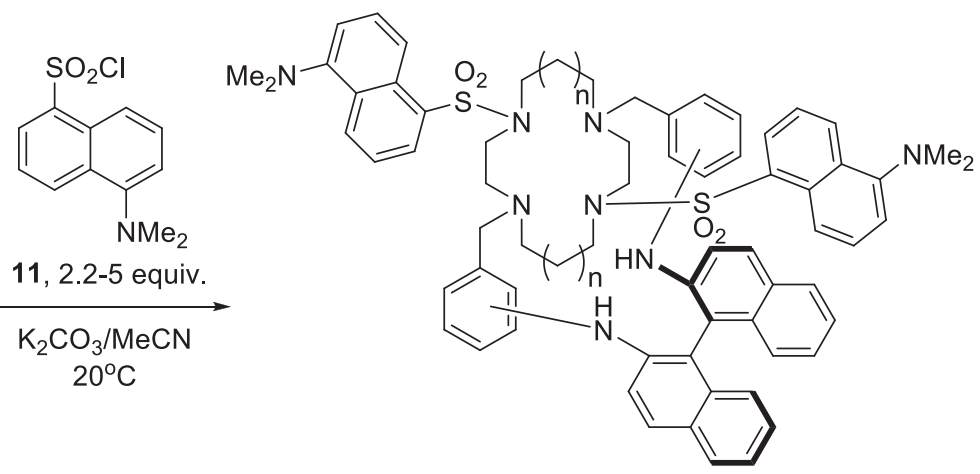

12: $\mathrm{n}=0, m-\mathrm{NH}, 90 \%$

13: $\mathrm{n}=0, p-\mathrm{NH}, 30 \%$

14: $n=1, m-\mathrm{NH}, 69 \%$ 


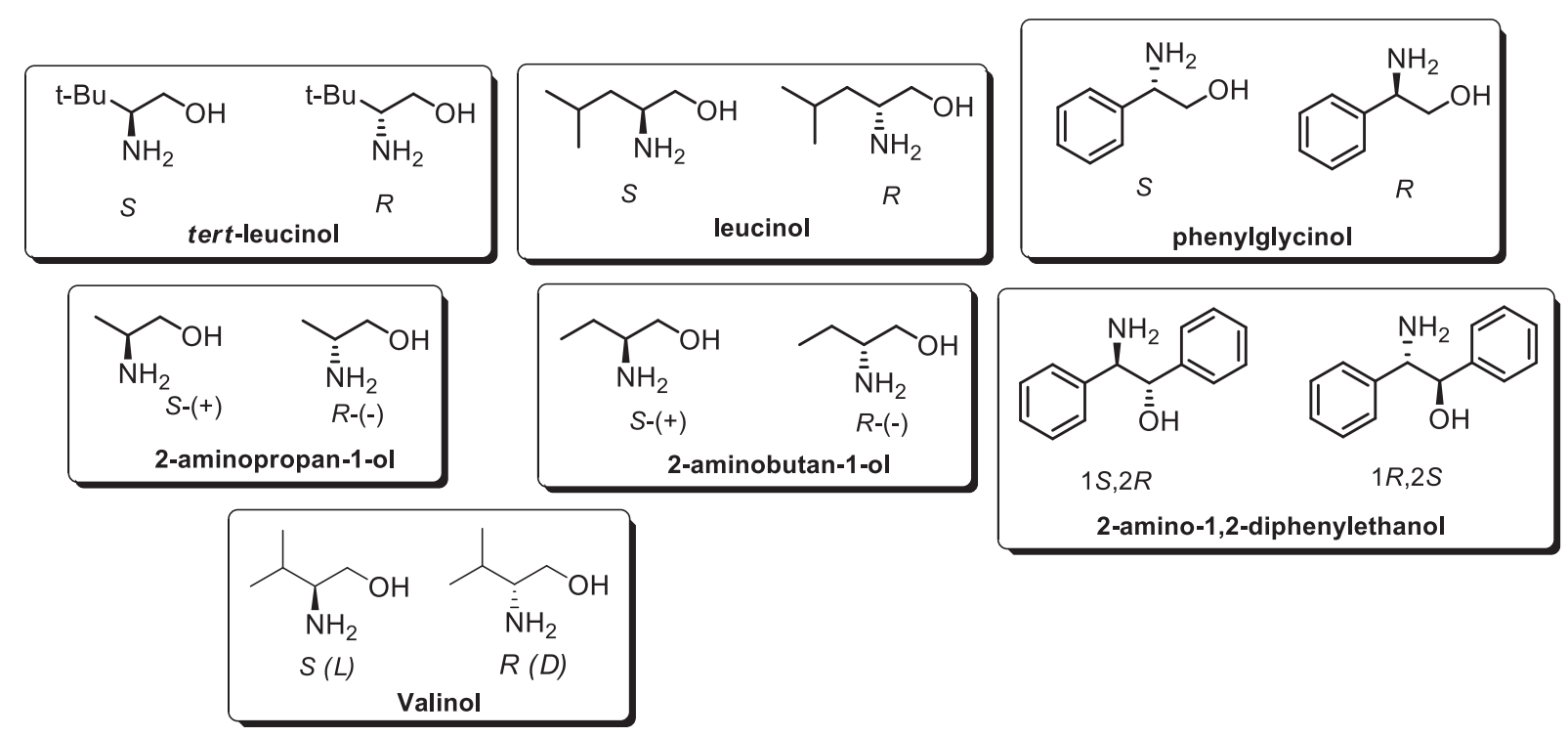

Figure 1. Optically active amino alcohols used in fluorescent detection experiments.

of BINAM are identical, as are the two dansyl groups in compound $\mathbf{1 4 .}$

Obtained cryptands were tested for their ability to perform as fluorescent enantioselective detectors using optically pure amino alcohols (7 pairs of enantiomers). The analytes structures are given on Figure 1. Experiments were carried out with the cryptands 6-9, and $\mathbf{1 3}$ in $\mathrm{MeCN}$, corresponding analytes were added stepwise (100, 200, 500,1000 equiv.) and fluorescent spectra were measured in each case. Compounds 6-9 possess an absorption band near $\lambda_{\text {max }}=350 \mathrm{~nm}$, and being excited at this wavelength they emit at $414 \mathrm{~nm}(\mathbf{6}), 432 \mathrm{~nm}$ (7), $422 \mathrm{~nm}(\mathbf{8}), 435 \mathrm{~nm}$ (9). The cryptand 6 did not change notably its fluorescence in the presence of both isomers of such amino alcohols like lecinol, tert-leucinol, 2-aminopropan-1-ol and 2-amino1,2-diphenyl ethanol. In the case of 2-aminobutan-1-ol, the addition of its $(S)$-isomer (here and after 1000 equiv. of each enantiomer are considered) quenched emission by $20 \%$ while $(R)$-isomer quenched it 1.5 times. Similarly, both isomers of phenylglycinol led to fluorescence quenching, $(S)$-isomer was more efficient (1.5 times) than $(R)$-isomer (by $40 \%$ ), moreover, in both cases bathochromic shift of the emission maxima by $20-25 \mathrm{~nm}$ was observed. Thus, compound 6 cannot be used for distinguishing between selected amino alcohols.

As for 7, the addition of enantiomers of the majority of amino alcohols also did not change notably the fluorescence intensity. In one case, with both $(S)$ - and $(R)$-isomers of 2-amino-1,2-diphenylethanol tiny quenching of emission was noted, and only the addition of the enantiomers of 2-phenylglycinol led to different results: with $(S)$-isomer the emission enhanced by $c$ a $20 \%$ while with $(R)$-isomer its intensity did not change (Figure 2). It makes possible to claim that the cryptand 7 is an efficient chemosensor for this amino alcohol.

The investigation of the compound $\mathbf{8}$ showed that the addition of both isomers of valinol resulted in the emission quenching: with $L$-isomer by $15 \%$, with $D$-isomer by $40 \%$. On the other hand, in the presence of both enan-

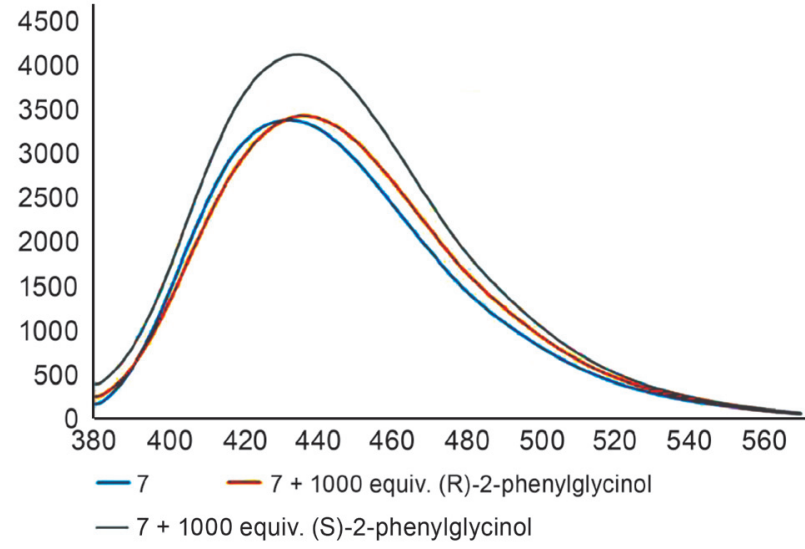

Figure 2. The spectra of fluorescence of the cryptand 7 in the presence of $(R)$ - and $(S)$-2-phenylglycinol (1000 equiv.).

tiomers of phenylglycinol or 2-amino-1,2-diphenyl ethanol emission enhancement was observed, more pronounced in the second case. The cryptand $\mathbf{8}$ turned to be unable to discriminate any of amino alcohols enantiomers.

The fluorescence of the cryptand 9 was susceptive to the presence of phenylglycinol enantiomers: the addition of its $(R)$-isomer led to an increase in the fluorescence intensity by $15 \%$ while $(S)$-isomer increased it by $40 \%$; simultaneously weak bathochromic shifts of the maxima ( $\mathrm{ca}$ $5-10 \mathrm{~nm}$ ) were noted. The addition of $L$-valinol caused a tiny emission quenching, and with $D$-valinol it was somewhat more pronounced. In fact, the compound 9 was found to be able to distinguish between 2-aminobutan-1-ol enantiomers: the presence of its $(S)$-isomer does not change the spectrum at all and its $(R)$-isomer causes $15 \%$ emission quenching (Figure 3). Thus, both cryptands 7 and 9 which were found to be more selective towards certain amino alcohols, comprise para-phenylene spacer.

Unfortunately, the modification of the macrobicycles with two dansyl fluorophores was not helpful and all amino 


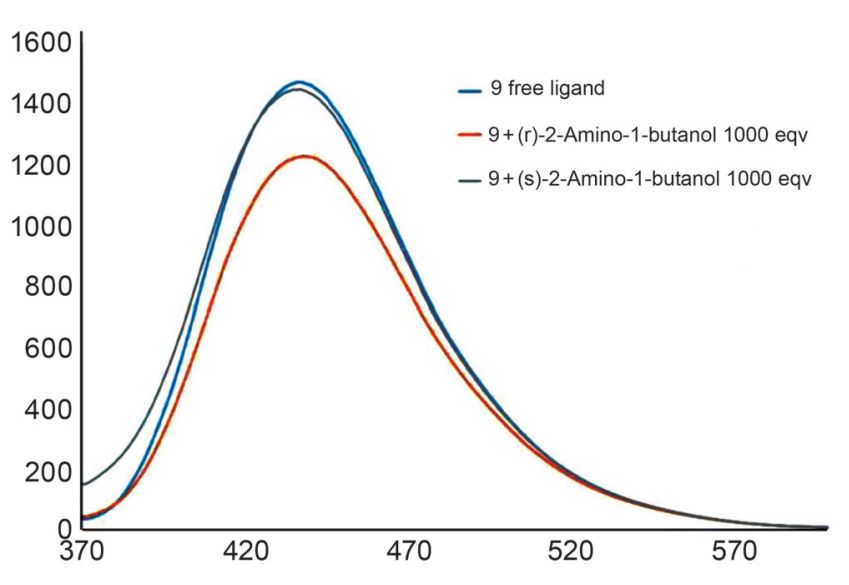

Figure 3. The spectra of fluorescence of the cryptand 9 in the presence of $(R)$ - and (S)-2-aminobutan-1-ol (1000 equiv.).

alcohols under investigation did not cause significant changes in the fluorescence intensity of compound $\mathbf{1 3 .}$ Probably, this is due to a strong shielding of the coordination sites with dimethylaminonaphthalene groups preventing the formation of the molecular complexes with even small organic compounds.

The fluorimetric investigations were continued with metal salts (also in $\mathrm{MeCN}$ ). Totally 21 metal cations were used for this purpose: $\mathrm{Li}^{\mathrm{I}}, \mathrm{Na}^{\mathrm{I}}, \mathrm{K}^{\mathrm{I}}, \mathrm{Mg}^{\mathrm{II}}, \mathrm{Ca}^{\mathrm{II}}, \mathrm{Ba}^{\mathrm{II}}, \mathrm{Al}^{\mathrm{III}}, \mathrm{Cr}^{\mathrm{III}}$, $\mathrm{Mn}^{\mathrm{II}}, \mathrm{Fe}^{\mathrm{II}}, \mathrm{Co}^{\mathrm{II}}, \mathrm{Ni}^{\mathrm{II}}, \mathrm{Cu}^{\mathrm{II}}, \mathrm{Zn}^{\mathrm{II}}, \mathrm{Cd}^{\mathrm{II}}, \mathrm{Pb}^{\mathrm{II}}, \mathrm{Ag}^{\mathrm{I}}, \mathrm{Hg}^{\mathrm{II}}$ (perchlorates), Ga ${ }^{I I I}$, In ${ }^{\mathrm{III}}$, Y ${ }^{\mathrm{III}}$ (nitrates). The addition of the metal salts led to much more pronounced changes in fluorescence with all cryptands tested. Some metals led to emission quenching, the most important effect was noted for $\mathrm{Hg}^{\mathrm{II}}$ (by $70 \%$ ) and especially by $\mathrm{Cu}^{\mathrm{II}}$ which produced full quenching (Figure S1, see Supporting Information at https://macroheterocycles.isuct.ru/en/mhc190337a). It was shown that the addition even of the first equivalent of this cation led to a dramatic decrease in the intensity of the emission (Figure S2). Some metals caused tiny emission enhancement, only in the presence of $\mathrm{In}^{\mathrm{III}}$ it increased substantially accompanied by a hypsochromic shift of the maximum by $10 \mathrm{~nm}$. It was shown that this shift was observed with the addition of the first equivalent of indium, and the enhancement was notable after the second equivalent was added (Figure S3). The maximal effect was achieved with 15 equiv. and further input of the salt did not change the spectrum. As for $\mathrm{Ga}^{\mathrm{III}}$, the maximal enhancement was noted for 5 equiv. of this metal while with 10 equiv. the intensity of fluorescence decreased to some extent (Figure S4). This effect may be explained by the formation of a new complex with different structure and spectroscopic features. Thus, the cryptand 6 can be considered as a molecular sensor for $\mathrm{Cu}^{\mathrm{II}}$ and $\mathrm{In}^{\mathrm{III}}$.

The fluorescence of the cryptand 7 also changes in the presence of most of the cations investigated (Figure $\mathrm{S} 5$ ): the addition of $\mathrm{Co}^{\mathrm{II}}$ and $\mathrm{Ni}^{\mathrm{II}}$ led to $\mathrm{ca}$ twofold decrease in the emission intensity while $\mathrm{In}^{\mathrm{III}}, \mathrm{Pb}^{\mathrm{II}}, \mathrm{Cr}^{\mathrm{III}}$, $\mathrm{Al}^{\mathrm{III}}, \mathrm{Ga}^{\mathrm{III}}$ and $\mathrm{Zn}^{\mathrm{II}}$ induced about twofold enhancement of the fluorescence intensity. These changes in emission were accompanied with small hypsochromic shifts (by less than $10 \mathrm{~nm}$ ) in the majority of cases. However, the addition of only 2 equiv. of $\mathrm{Cu}^{\mathrm{II}}$ quenched totally the fluorescence
(Figure S6), this can be helpful in the selective detection of this metal.

The addition of the metal salts to the macrobicycle 8 generally results in more or less pronounced emission quenching (Figure S7). The most efficient quenching was noted for $\mathrm{Hg}^{\mathrm{II}}$ (by $45 \%$ ), $\mathrm{Co}^{\mathrm{II}}$ (by $60 \%$ ) and $\mathrm{Cu}^{\mathrm{II}}$ (by $80 \%$ ). It is clearly seen from Figure S8 that unlike in the case of the two previous ligands 6 and 7, here the addition of even 10 equiv. of copper was not enough to achieve full emission quenching. However, with this metal cation the hypsochromic shift of the emission maximum from 422 to $406 \mathrm{~nm}$ was observed. This effect can distinguish copper among other metal cations. No enhancement of the fluorescence intensity was noted in any case, unlike it was common for cyclen-based cryptands 6 and 7.

As for the cryptand 9, most of metal cations more or less diminished the intensity of fluorescence. Three of them, $\mathrm{Hg}^{\mathrm{II}}, \mathrm{Cd}^{\mathrm{II}}$ and $\mathrm{Cu}^{\mathrm{II}}$ led to hypsochroomic shifts of the emission maxima (by 5, 10 and $25 \mathrm{~nm}$, respectively) (Figure S9). In the presence of these metals fluorescence quenched by 70,65 and $85 \%$, respectively. Almost full emission quenching with $\mathrm{Cu}^{\mathrm{II}}$ was achieved with 10 equiv. (Figure S10). The macrobicycle 9 can be judged as a molecular probe for the above-mentioned metals. To note, cyclen-containing cryptands $\mathbf{6}$ and $\mathbf{7}$ undergo almost full emission quenching in the presence of 2-5 equiv. of $\mathrm{Cu}^{\mathrm{II}}$ cations, while the fluorescence of cyclam-containing macrobicycles $\mathbf{8}$ and $\mathbf{9}$ is quenched only partially by this metal added in comparable amounts. This fact is in a good correspondence with the known better stability of the cyclencopper complex compared to cyclam analogue.

Quite different behavior of fluorescence in the presence of the studied metals was observed in the case of the dansyldecorated cryptand 13 (Figure S11). Excitation of the ligand at $340 \mathrm{~nm}$ led to intensive emission at $512 \mathrm{~nm}$. No enhancement of the emission was noted in the presence of any ligand, and many metals caused partial quenching of fluorescence. Its intensity diminished by $20-40 \%$ in the presence of $\mathrm{Al}^{\mathrm{III}}$, $\mathrm{In}^{\mathrm{III}}, \mathrm{Y}^{\mathrm{III}}, \mathrm{Fe}^{\mathrm{II}}, \mathrm{Ni}^{\mathrm{II}}, \mathrm{Zn}^{\mathrm{II}}, \mathrm{Pb}^{\mathrm{II}}$, and $\mathrm{Cu}^{\mathrm{II}}$ led to 5-times decrease in the emission intensity. Moreover, generally bathochromic shifts of the emission maxima were observed (525-535 nm) after the addition of metal salts which caused quenching. To note, full emission quenching by the copper cations was achieved after adding 10 equiv. of the corresponding salt (Figure S12).

\section{Conclusions}

To conclude, in the course of the present work we used our previously elaborated method for the synthesis of BINAMcontaining macrobicycles based on disubstituted cyclen and cyclam. The compounds were decorated with dansyl fluorophore groups. Spectrofluorimetric investigations of synthesized cryptands demonstrated the possibility of the cyclen-based compound 7 to distinguish between two enantiomers of 2-phenylglycinol and of cyclam-based macrobicycle 9 to detect enantiomers of 2-aminobutan-1-ol by different changes in the emission caused by the presence of enantiomers of said compounds. Spectrofluorimetric titrations of the ligands revealed the ability of the cryptand 7 
to act as a selective fluorescent detector of $\mathrm{Cu}^{\mathrm{II}}$ cations, while macrobicycles $6,8,9$, and 13 were shown to be molecular sensors for $\mathrm{Cu}^{\mathrm{II}}, \mathrm{Hg}^{\mathrm{II}}, \mathrm{Cd}^{\mathrm{II}}$ and $\mathrm{In}^{\mathrm{III}}$.

Acknowledgements. The authors acknowledge the financial support of the RFBR grants 18-03-00709 and 17-53-16012.

\section{References}

1. Iwanek W., Mattay J.J. Photochem. Photobiol. A 1992, 67, 209.

2. Xu K., Qiu Z., Zhao J., Zhao J., Wang C. Tetrahedron: Asymmetry 2009, 20, 1690.

3. Xu K., Yang L., Wang Y., Zhao J., Wang C. Supramol. Chem. 2010, 22, 563.

4. Lin J., Hu Q., Xu M., Pu L. J. Am. Chem. Soc. 2002, 124, 2088.

5. Xu M., Lin J., Hu Q., Pu L. J. Am. Chem. Soc. 2002, 124, 14239.

6. Lin J., Zhang H., Pu L. Org. Lett. 2002, 4, 3297.

7. Li Z., Lin J., Zhang H., Sabat M., Hiacinth M., Pu L. J. Org. Chem. 2004, 69, 6284

8. Xu K., Jiao S., Yao W., Xie E., Tang B., Wang C. Chirality 2012, 24, 646.

9. Bencini A., Coluccini C., Garau A., Giorgi C., Lippolis V., Messori L., Pasini D., Puccioni S. Chem. Commun. 2012, 48, 10428.

10. Luo J., Zheng Q., Chen C., Huang Z. Tetrahedron 2005, 61, 8517.

11. Wang H., Tian X., Yang D., Pan Y., Wu Q., He C. Tetrahedron: Asymmetry 2011, 22, 381.

12. Cho E.N.R., Li Y., Kim H.J., Hyun M.H. Chirality 2011, 23, 349.

13. Kim K.S., Jun E.J., Kim S.K., Choi H.J., Yoo J., Lee C., Hyun M.H., Yoon J. Tetrahedron Lett. 2007, 48, 2481.

14. Ema T., Ura N., Eguchi K., Ise Y., Sakai T. Chem. Commun. 2011, 47, 6090 .
15. Ema T., Ura N., Eguchi K., Sakai T. Bull. Chem. Soc. Jpn. 2012, 85, 101.

16. Parker K.S., Townshend A., Bale S. J. Anal. Commun. 1996, $33,265$.

17. Murakoshi K., Azechi T., Hosokawa H., Wada Y., Yanagida S. J. Electroanal. Chem. 1999, 473, 117.

18. Wei L., He Y., Xu K., Liu S., Meng L. Chin. J. Chem. 2005, 23,757 .

19. Meng J., Wei G., Huang X., Dong Yu., Cheng Y., Zhu C. Polymer 2011, 52, 363.

20. Grigorova O.K., Averin A.D., Maloshitskaya O.A., Beletskaya I.P. Macroheterocycles 2016, 9, 425.

21. Grigorova O.K., Averin A.D., Maloshitskaya O.A., Beletskaya I.P. Macroheterocycles 2017, 10, 446.

22. Grigorova O.K., Gusev D.I., Averin A.D., Maloshitskaya O.A., Beletskaya I.P. Russ. Chem. Bull. 2019, 848.

23. Ukai T., Kawazura H., Ishii Y., Bonnet J.J., Ibers J.A. $J$ Organomet. Chem. 1974, 65, 253.

24. Averin A.D., Shukhaev A.V., Buryak A.K., Denat F., Guilard R., Beletskaya I.P. Tetrahedron Lett. 2008, 49, 3950.

25. Kobelev S.M., Averin A.D., Buryak A.K., Denat F., Guilard R., Beletskaya I.P. Heterocycles 2011, 82, 1447.

26. Kobelev S.M., Averin A.D., Buryak A.K., Savelyev E.N., Orlinson B.S., Butov G.M., Novakov I.A., Denat F., Guilard R., Beletskaya I.P. ARKIVOC 2012, vii, 196.

27. Averin A.D., Tyutenov K.S., Shukhaev A.V., Kobelev S.M., Buryak A.K., Denat F., Guilard R., Beletskaya I.P. Heterocycles 2012, 86, 1341.

28. Kobelev S.M., Averin A.D., Maloshitskaya O.A., Denat F., Guilard R., Beletskaya I.P. Macroheterocycles 2012, 5, 389.

29. Averin A.D., Shukhaev A.V., Vovk A.I., Kukhar V.P., Denat F., Guilard R., Beletskaya I.P. Macroheterocycles 2014, 7, 174.

30. Kobelev S.M., Averin A.D., Buryak A.K., Denat F., Guilard R., Beletskaya I.P. Macroheterocycles 2014, 7, 28.

31. Kobelev S.M., Averin A.D., Buryak A.K., Vovk A.I., Kukhar V.P., Denat F., Guilard R., Beletskaya I.P. Heterocycles 2015, 90, 989. 